\title{
Cesarean section after abdominal mesh repair for pregnancy-related desmoid tumor: a case report
}

This article was published in the following Dove Press journal:

International Journal of Women's Health

12 July 2017

Number of times this article has been viewed

\section{Sara Ooi \\ Harry Ngo}

Obstetrics and Gynaecology Department, Liverpool Hospital, Liverpool, NSW, Australia
Correspondence: Harry Ngo Suite 8/I-5 Jacobs Street, Bankstown, NSW 2200, Australia

Tel +6I 297072488

Email harryanghango@gmail.com

\begin{abstract}
We report the case of a 32-year-old gravida 2 para 1 woman with a background of partially resected desmoid tumor (DT) arising from the previous cesarean section (CS) scar. This case details the management of her DT by surgical resection and mesh repair and second pregnancy following this. Pregnancy-related DTs are a relatively rare entity, and there is a paucity of literature regarding their management during pregnancy. There are only five reported cases of DTs arising from CS scars. To our knowledge, this is the only report to illustrate that subsequent $\mathrm{CS}$ is possible after desmoid resection and abdominal mesh repair. It provides evidence that CS can be safely accomplished following abdominal wall reconstructions and further arguments against elective lower segment CS.
\end{abstract}

Keywords: abdominal wall, cesarean section, complications, desmoid tumor, surgical mesh

\section{Case presentation}

The patient a 32-year-old gravida 2 para 1 (G2P1) Vietnamese woman presented to a private obstetric consulting room for antenatal management of her second pregnancy in, May 2014.

The patient had an elective lower segment cesarean section (LSCS) during her first pregnancy in 2009, and since then, noted gradually increasing "hardness" in her wound and worsening wound pain. In 2011, she presented to obstetric consulting room for further investigation, and on examination, she was found to have a mildly tender, firm $3 \mathrm{~cm}$ lump at the left lateral aspect of her cesarean section (CS) scar. Pelvic ultrasound revealed a heterogeneous $3.3 \times 3.4 \times 1.7 \mathrm{~cm}$ mass, thought to be an incisional hernia. Computed tomography (CT) of abdomen showed a "diffuse thickening of the left rectus abdominis muscle in the lower abdomen, without any focal mass or fluid collection", excluding incisional hernia. Differential diagnoses included muscle hematoma, chronic inflammation and postsurgical desmoid or endometriosis. There was no history of blunt trauma to the area.

In late 2011, the patient underwent surgical wound exploration involving left rectus muscle incision and partial lump excision. She had a good recovery postoperatively and noted a significant improvement in her pain. Histopathology of the macroscopically firm and rubbery tissue revealed spindle cell proliferation infiltrating adjacent skeletal muscle and adipose tissue on a background of small vascular proliferation. These features were consistent with an extra-abdominal desmoid-type fibromatosis. The lesion extended to the surgical margins multifocally, and follow-up CT showed a significant amount of residual desmoid in the lower abdominal wall.

In 2012, the patient expressed wishes to have another child. Considering the hormone-sensitive nature of desmoid tumors (DTs) and the high chance of substantial 
growth of the remaining desmoid tissue during pregnancy, it was decided that the residual tissue should be removed prior to pregnancy. The DT was fully resected, requiring anterior rectus muscle reconstruction with mesh. Intraoperatively, the inferior epigastric vein was found to be entwined among the tumor and required reconstruction at its attachment to the external iliac vein by the vascular team.

When the patient presented to obstetric consulting room in May 2014, she was at 8 weeks gestation. Her pregnancy was progressing well, with unremarkable antenatal screening. She was healthy and well, with no significant medical history and no medications apart from folate and iron supplementation. After discussion with the surgical team who performed the mesh repair, it was decided that the patient would undergo a repeat CS followed by reapproximation of the divided mesh.

In early 2015, the patient presented to a hospital in developed labor at 39 weeks gestation. She had an emergency LSCS, involving dividing the anterior abdominal mesh and reapproximating this with nonabsorbable sutures, followed by standard rectus sheath closure. There was a significant adhesion of the mesh to underlying tissue, leading to subsequent difficult entry into the peritoneum. The patient delivered a healthy boy with birth weight 3,158 $\mathrm{g}$ and Apgar scores 9 and 9 (at 1 and 5 minutes, respectively). She made an uneventful recovery, with good healing of her surgical wound. She has had no clinically identifiable desmoid recurrence to date. The patient has consented to publication of this report.

\section{Discussion and literature review}

DTs are rare, locally invasive but benign lesions of fibroblastic origin. Incidence is reported to be two to four people affected per $1,000,000,{ }^{1}$ with a slight female predominance, although this varies throughout the literature. ${ }^{2}$ Clinical presentation and natural history vary considerably and are still incompletely understood. Pregnancy-related DTs specifically are those that arose, were discovered or grew during pregnancy, or developed postpartum within 36 months. ${ }^{3}$ They most commonly occur within the rectus abdominis muscle, ${ }^{4}$ although cases have been reported of various other locations, including the vulva $^{5}$ and larynx. ${ }^{6}$ They are commonly initially mistaken for incisional hernias, ${ }^{7}$ as was the case with our patient.

Despite their rarity and benign nature, they are clinically relevant and warrant further research. Local invasion or compression of nearby structures can lead to significant morbidity, for example, persistent unstable fetal lie $^{8}$ or pain and dyspnea. ${ }^{9}$

The etiology of DTs also remains uncertain, although it is likely multifactorial. Associations have been made between
DTs and familial adenomatous polyposis, ${ }^{10}$ hormonal states (potentially explaining increased tumor growth in pregnancy) $)^{11}$ and surgical or blunt trauma. ${ }^{12}$ Our patient had no known personal or family history or Familial Adenomatous Polyposis (FAP); her risk factors for desmoid occurrence include combined oral contraceptives and previous CS.

A search of the literature revealed only four reported cases of DTs arising from a cesarean scar. ${ }^{13-16}$ In one case, as reported by Cian et al, ${ }^{16}$ the desmoid was monitored throughout the pregnancy with no increase in size during 6 months gestation and removed with mesh repair at the time of delivery.

The literature provides little information regarding how to counsel women who have had a DT and wish to have further children. Our patient's case is the first reported in the literature of a subsequent pregnancy being delivered by CS through a reconstructed abdominal wall. In a multi-institutional analysis of 92 women across four sarcoma centers, Fiore et $\mathrm{al}^{17}$ found that progression risk during pregnancy is high, although it can be safely managed. For our patient, the decision was made to remove the residual desmoid tissue prior to conception to alleviate this risk.

The management of DTs, regardless of pregnancy status, is primarily surgical; however, there is a clear lack of Level 1 evidence regarding the best practice. The recommended treatment is wide surgical excision if possible, with resection margins possibly correlating to recurrence rate. ${ }^{18,19}$ When positive or close surgical margins are likely, adjuvant or definitive radiotherapy is preferred. ${ }^{20}$ Sutton and Thomas ${ }^{21}$ reported a case series of seven patients treated by wide surgical excision and mesh reconstruction, in a similar method as in our patient, with all but one patient having no tumor recurrence at the median follow-up time of 3.5 years. Other management options include chemotherapeutic agents, nonsteroidal anti-inflammatory drugs, or antiestrogenic agents such as tamoxifen. ${ }^{18}$ Fine needle aspirate or core needle biopsy may also be useful in preoperative diagnosis. ${ }^{22}$

As this case is the only report to describe successful CS post-desmoid resection and abdominal repair, it provides unique insights into the management of pregnant women with prior desmoid resection.

The literature provides little information regarding how to counsel women who have had a DT and wish to have further children. This case shows that subsequent pregnancy is achievable and safe following resection of some abdominal wall tumors. It also provides further evidence that CS can be safely accomplished following abdominal wall reconstructions. 


\section{Disclosure}

The authors report no conflicts of interest in this work.

\section{References}

1. Molelekwa V, Fernandes H, Kerin MJ, Geary M. Conservative management of a desmoid tumour in pregnancy. J Obstet Gynaecol. 2004; 24(6):700.

2. Anonymous. The desmoid tumour. Br Med J. 1969;4(5681):447-448.

3. Robinson W, McMillan C, Kendall A, Pearlman N. Desmoid tumors in pregnant and postpartum women. Cancer. 2012;4(1):184-192.

4. Gansar GF, Markowitz IP, Cerise EJ. Thirty years of experience with desmoid tumors at Charity Hospital. Am Surg. 1987;53(6):318-319.

5. Allen MV, Novotny DB. Desmoid tumor of the vulva associated with pregnancy. Arch Pathol Lab Med. 1997;121(5):512-514.

6. Gherman RB, Bowen E, Eggleston MK, Karakla D. Desmoid tumor of the larynx complicating pregnancy: a case report. Am J Obstet Gynecol. 1999;180(4):1036-1037.

7. Khoo SK. The desmoid tumor, a diagnostic problem in the gynaecological patient: a report of four patients. Aust N Z J Surg. 1972; 42(2):170-174.

8. Kumar R, Lynch P. Large desmoid tumour causing unstable lie in pregnancy. J Obstet Gynaecol. 2012;32(4):395-396.

9. Zhou H, Lu H, Wang L, et al. Abdominal wall desmoid tumor during pregnancy: case report and literature review. Clin Exp Obstet Gynecol. 2015;42(2):253-258.

10. Mulik V, Griffiths AN, Beattie RB. Desmoid tumours with familial adenomatous polyposis in pregnancy. J Obstet Gynaecol. 2003;23(3): 307-308.

11. Durkin AJ, Korkolis DP, Al-Saif O, Zervos EE. Full-term gestation and transvaginal delivery after wide resection of an abdominal desmoid tumor during pregnancy. J Surg Oncol. 2005;89(2):86-90.
12. Firoozmand E, Prager E. Pelvic desmoid tumor: threat to mother and fetus. Am Surg. 2001;67(12):1213-1215.

13. Herman K, Marcinek A. Desmoid powloki brzusznej u 28-letniej ciezarnej. [Abdominal desmoid in a 28-year old pregnant woman]. Ginekol Pol. 1996;67(7):374-375. Polish.

14. Sharp N, Daw E. Desmoid tumor in a cesarean section scar. Am JObstet Gynecol. 1984;149(1):83.

15. Brezinka C, Huter O, Dietze O, Steiner H. Desmoidtumor in einer Sectionarbe als Geburtshindernis bei erneuter Schwangerschaft. [Desmoid tumor in a cesarean section scar as a labor complication in subsequent pregnancy]. Geburtshilfe Frauenheilkd. 1986;46(12): 908-909. German.

16. De Cian F, Delay E, Rudigoz RC, Ranchere D, Rivoire M. Desmoid tumor arising in a cesarean section scar during pregnancy: monitoring and management. Gynecol Oncol. 1999;75(1):145-148.

17. Fiore M, Coppola S, Cannell AJ, et al. Desmoid-type fibromatosis and pregnancy: a multi-institutional analysis of recurrence and obstetric risk. Ann Surg. 2014;259(5):973-978.

18. Way JC, Culham BA. Desmoid tumour. The risk of recurrent or new disease with subsequent pregnancy: a case report. Can J Surg. 1999; 42(1):51-54.

19. Zingo L, Cusumano F, Salvadori B. Abdominal and extra-abdominal desmoid tumors. Report of 21 cases. Tumori. 1980;66(1):101-107.

20. Joglekar S, Rose PS, Sim F, Okuno S, Peterson I. Current perspectives on desmoid tumors: the mayo clinic approach. Cancers (Basel). 2011; 3(3):3143-3155.

21. Sutton RJ, Thomas JM. Desmoid tumours of the anterior abdominal wall. Eur J Surg Oncol. 1999;25(4):398-400.

22. Dalén BPM, Meis-Kindblom JM, Sumathi VP, Ryd W, Kindblom L-G. Fine-needle aspiration cytology and core needle biopsy in the preoperative diagnosis of desmoid tumors. Acta Orthop. 2006;77(6): 926-931.
International Journal of Women's Health

\section{Publish your work in this journal}

The International Journal of Women's Health is an international, peerreviewed open-access journal publishing original research, reports, editorials, reviews and commentaries on all aspects of women's healthcare including gynecology, obstetrics, and breast cancer. The manuscript management system is completely online and includes

\section{Dovepress}

a very quick and fair peer-review system, which is all easy to use. Visit http://www.dovepress.com/testimonials.php to read real quotes from published authors. 